\title{
Ureilite meteorites provide a new model of early planetesimal formation and destruction
}

\author{
N. Rai ${ }^{1,2,3^{*}}$, H. Downes ${ }^{2,3}$, C. Smith ${ }^{3}$
}

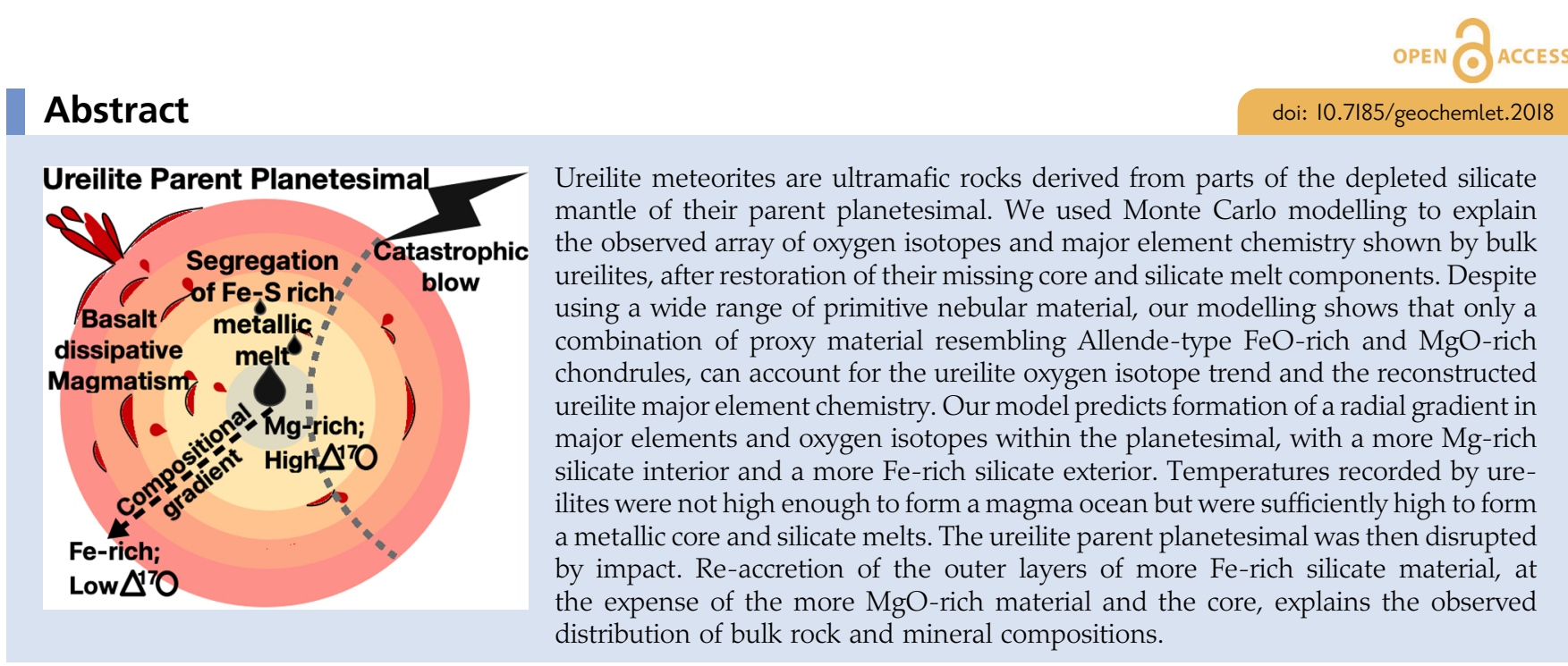

Received 11 February 2019 | Accepted 6 May 2020 | Published 8 July 2020

\section{Introduction}

Planetesimals represent an important stage in the formation of terrestrial planets (Elkins-Tanton, 2012). Ureilite meteorites are mostly fragments of the silicate mantle of a differentiated planetesimal (Scott et al., 1993; Mittlefehldt et al., 1998). They are a common group of achondrites and their existence must be explained in any comprehensive model of planetesimal formation. Here we present a model for accretion, differentiation and destruction of the ureilite planetesimal, which accounts for oxygen isotope compositions, major element compositions, and trace element abundances.

Ureilite meteorites provide a window into major planetesimal-forming processes. They are carbon-bearing, olivine-dominated meteorites (Mittlefehldt et al., 1998) which are mostly unbrecciated but also occur as polymict breccias (Goodrich et al., 2004). Almahata Sitta is a meteorite containing ureilitic clasts of different compositions and textures (Jenniskens et al., 2009; Goodrich et al., 2015). It was derived from a daughter asteroid which re-accreted after the original ureilite planetesimal was disrupted by impact (Herrin et al., 2010), thus preserving ureilite planetesimal material which might otherwise have been lost. The original ureilite planetesimal did not survive long enough to accrete into a terrestrial planet. However, it reached a size and temperature sufficient to enable it to undergo several important processes including partial melting and onset of core formation (Warren et al., 2006; Barrat et al., 2015).

Olivines in ureilites are often strongly zoned with high $\mathrm{MgO}$ rims, ascribed to late stage reduction during impact (Warren, 2012). Therefore, in this study, only mineral core compositions are discussed. Accessory carbon phases (up to 7 wt. \%), metals and sulfides are also present. Basaltic melt clasts in polymict ureilites are the remains of silicate magmas produced by partial melting of the parent planetesimal (Cohen et al., 2004). A trachyandesitic clast from Almahata Sitta also has oxygen isotopes and mineral compositions relating it to the ureilite planetesimal (Bischoff et al., 2014). Therefore, a range of silicate magmas from basalt to trachyandesite were produced by partial melting of the parent ureilite planetesimal.

Ureilitic olivine cores have core compositions from $\mathrm{FO}_{75}$ to $\mathrm{Fo}_{97}$ (Goodrich et al., 2004; Downes et al., 2008). Low Ca pyroxenes and augite are the second and third most abundant minerals, although rare ureilites are pyroxene-rich. Ureilites show a wide array of oxygen isotope compositions (Clayton and Mayeda, 1988), which fall close to the Carbonaceous Chondrite Anhydrous Mineral (CCAM) line and show a strong negative correlation with the Fo content of olivine and hence with bulk compositions of ureilites (Fig. 1). Interpretations of this correlation are contentious, but they strongly suggest that two component mixing caused much of the compositional variation

\footnotetext{
1. Dept of Earth Sciences, Indian Institute of Technology, Roorkee 247667, India

2. Dept of Earth and Planetary Sciences, Birkbeck University of London, Malet Street, London WC1E 7HX, UK

3. Dept of Earth Sciences, Natural History Museum, Cromwell Road, London SW7 5BD, UK

Corresponding author (email: n.rai@es.iitr.ac.in)
} 

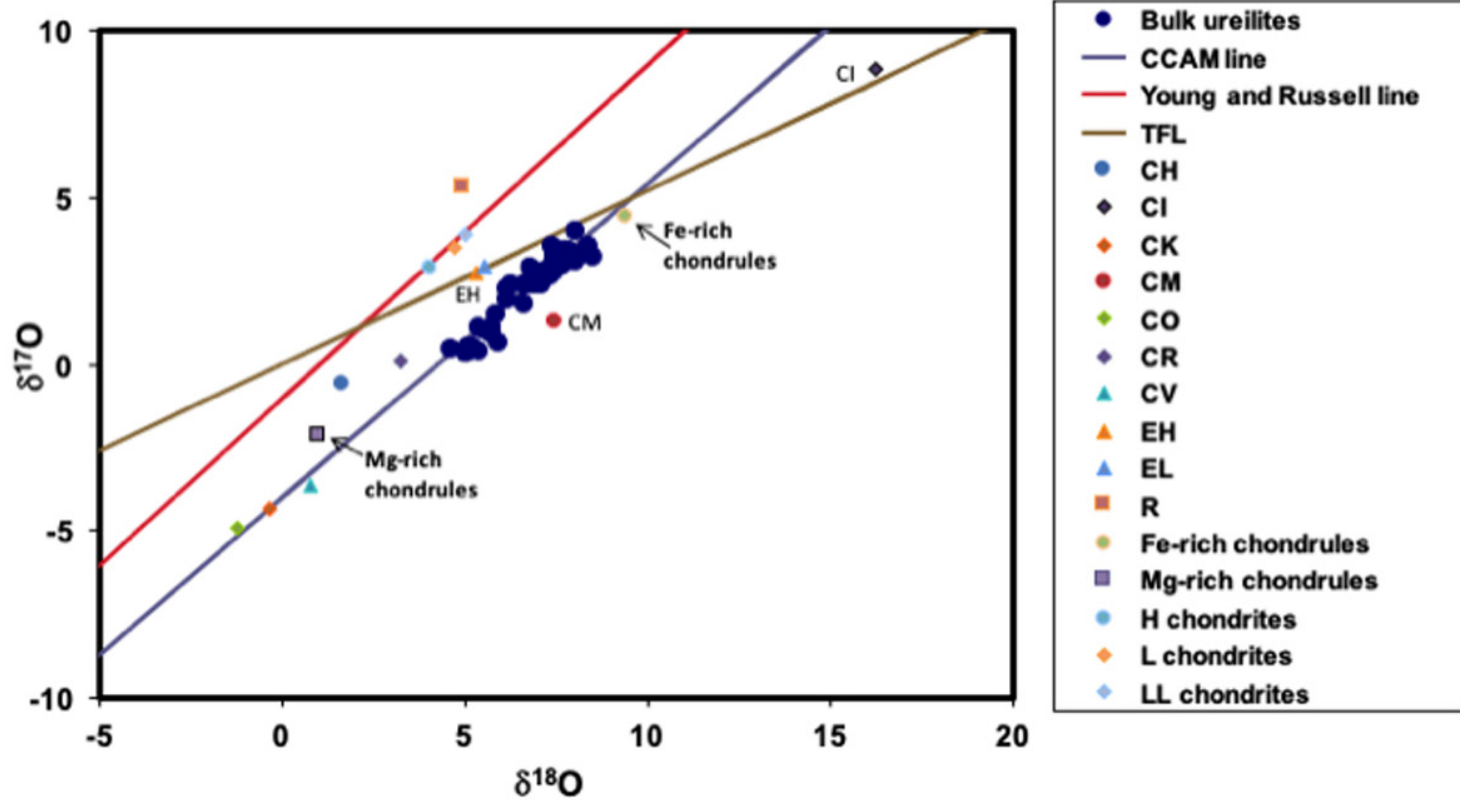

(a)

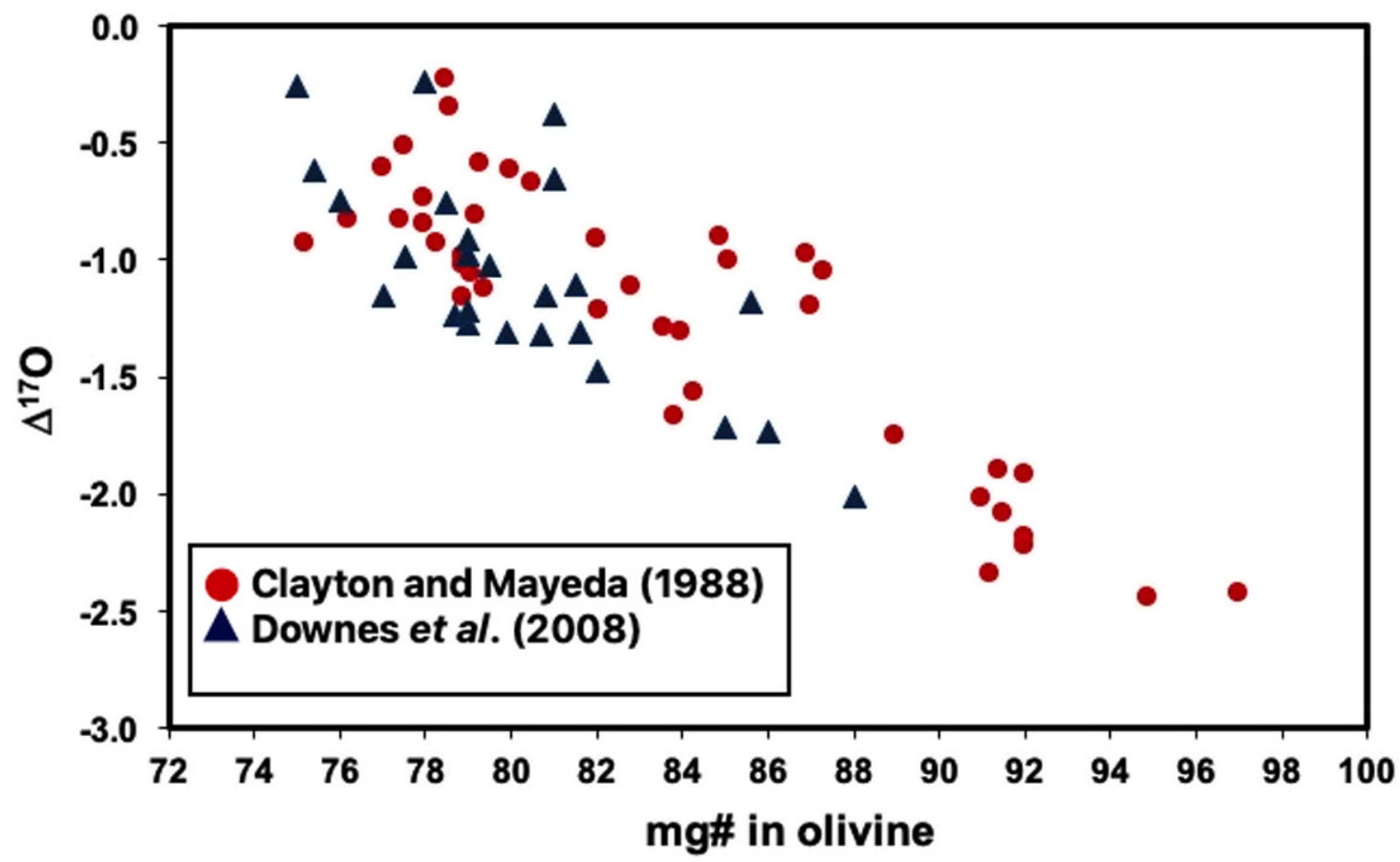

(b)

Figure 1 (a) Oxygen 3-isotope plot for bulk ureilites and ureilite clasts in polymict meteorites. (b) Bulk rock oxygen isotopes vs. Fo value in olivine cores.

(Sanders et al., 2017). Barrat et al. (2017) also showed that ureilite carbon formed from mixing of two different reservoirs, one with high $\delta^{13} \mathrm{C}$ and low $\mathrm{MgO}$ silicates, the other with low $\delta^{13} \mathrm{C}$ and more $\mathrm{MgO}$-rich silicates.

Figure 2 shows the distribution of ureilite olivine core compositions (Goodrich et al., 2004; Downes et al., 2008). Olivines with $\mathrm{FO}_{>85}$ are much less abundant than those of $\mathrm{Fo}_{<85}$. Any model of the present day parent body must explain the preponderance of $\mathrm{FeO}$-rich ureilites over $\mathrm{MgO}$-rich ones. Another significant correlation in ureilites is between olivine Fo values and calculated equilibrium temperature (Singletary and Grove, 2006; Warren, 2012). MgO-rich ureilites reached higher temperatures $\left(1320^{\circ} \mathrm{C}\right)$ than FeO-rich ones $\left(1190{ }^{\circ} \mathrm{C}\right)$. This correlation must be explained by any model of the original ureilite planetesimal.

\section{Results}

The anti-correlation between oxygen isotope compositions and the Fo content of constituent olivine in ureilites (Fig. 1b) indicates that a range of bulk ureilite compositions from $\mathrm{MgO}$-rich to FeO-rich must be modelled. We have therefore 


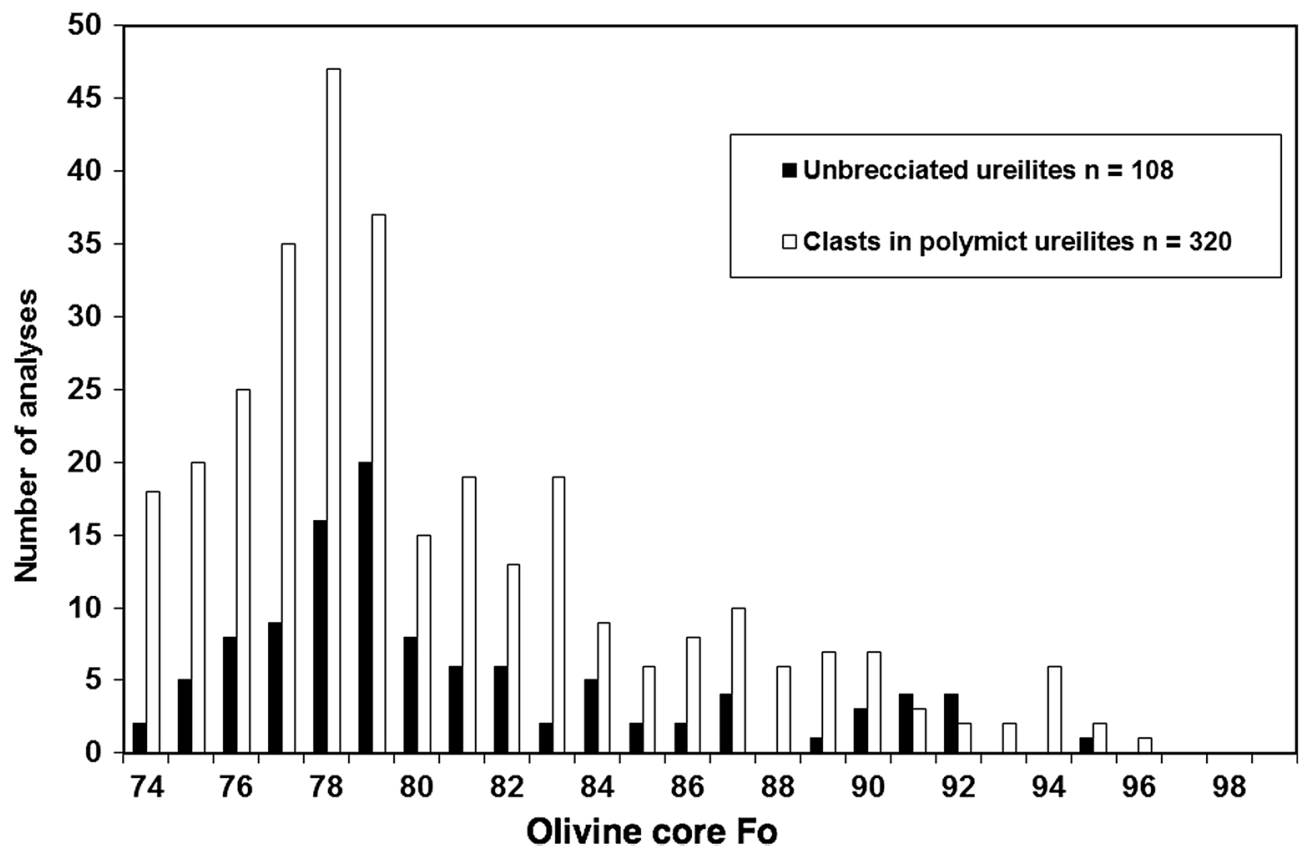

Figure 2 Histogram of olivine core compositions in monomict ureilites and ureilitic fragments in polymict ureilites (Goodrich et al., 2004; Downes et al., 2008).

modelled accretion of the original ureilite planetesimal using a range of oxygen isotopes and bulk major element compositions. Ureilites provide an estimate of the bulk mantle composition of their parent planetesimal after removal of a core and various silicate melts. These compositional constraints are provided by the metal- and sulfide-free ureilite data of Warren (2012) (Table S-1, see Supplementary Information).

We used Monte Carlo simulations to calculate combinations of different chondritic components (Table S-1), together with $\mathrm{FeO}$-rich and $\mathrm{MgO}$-rich chondrules. The chondrules used for modelling are a proxy for the real primitive materials that formed the original parent body, which was probably formed from an earlier generation of chondrules rarely seen in meteorites (Libourel and Krot, 2007). We ran 100,000 Monte Carlo simulations to assess two, three and four end member combinations of all components that could simultaneously satisfy the oxygen isotope characteristics $\left(\Delta^{17} \mathrm{O}, \delta^{18} \mathrm{O}\right.$ and $\left.\delta^{17} \mathrm{O}\right)$. For each simulation, we allowed the proportions of components to vary randomly (maintaining a total of $100 \%$ ) and determined whether the resulting oxygen isotope characteristics matched those of ureilites. We found that no model consisting entirely of bulk chondrites and/or CAIs could satisfy the oxygen isotope criteria. We did not find any valid three or four end member solutions that could exactly reproduce the entire trend of ureilite oxygen isotopes without over-generating or under-generating the range of observed ureilite oxygen isotopes. The only possible solution is a binary mixture consisting of $\mathrm{FeO}$-rich and $\mathrm{MgO}$-rich chondrules that can reproduce the entire trend of ureilite oxygen isotopes. The ureilite compositional range is bracketed by two end member mixtures: (i) 92 wt. \% FeO-rich chondrules with 8 wt. \% $\mathrm{MgO}$-rich chondrules and (ii) 33 wt. \% FeO-rich chondrules with 67 wt. \% MgO-rich chondrules.

We then evaluated the range of bulk ureilite major element compositions predicted by this combination. Figure 3 shows that the range of metal-free ureilite compositions has $\mathrm{Fe} / \mathrm{Si}$ and $\mathrm{Al} / \mathrm{Si}$ ratios which are too low to be accounted for by any combination of chondrules, and $\mathrm{Mg} / \mathrm{Si}$ ratios which are higher than all bulk chondritic material. This clearly indicates that a missing silicate melt needs to be added back to restore the original bulk composition of the ureilite planetesimal. Our models used several silicate melt compositions (Table S-1). We found that, for $\mathrm{Mg} / \mathrm{Si}, \mathrm{Al} / \mathrm{Si}$ and $\mathrm{Ca} / \mathrm{Al}$ ratios, the reconstructed bulk ureilite compositions can be matched by the addition of $5 \%$ basalt to $95 \%$ depleted ureilite mantle, regardless of Mg\# of the mantle.

However, the $\mathrm{Fe} / \mathrm{Si}$ and $\mathrm{Fe} / \mathrm{Al}$ ratios of the model bulk compositions could not be matched, because we did not account for segregation of an Fe-rich core. Adding a core of 1-6 \% FeS elevated the $\mathrm{Fe} / \mathrm{Si}$ and $\mathrm{Fe} / \mathrm{Al}$ ratios and matched the model values. Thus, our two end member chondrule model predicts a $\mathrm{FeS}$ core consistent with observed siderophile element systematics of ureilites (Rankenburg et al., 2008; Hayden et al., 2011; Warren, 2012). It also predicts that a "ureilite chondrite" existed which was composed of mostly high $\mathrm{FeO}$ chondrules with a minor amount of $\mathrm{MgO}$-rich chondrules, analogous to the "lodranite chondrite" reported by Li et al. (2018).

\section{Discussion}

Any model of the evolution of the ureilite planetesimal must account for the range of bulk compositions and the strong correlations in oxygen isotopes in ureilite meteorites (Figs. 1, 3), the differences in temperature shown by $\mathrm{MgO}$-rich and $\mathrm{FeO}-$ rich ureilites, and the observed distribution of olivine core Fo contents (Fig. 2). We propose a series of stages, leading to the formation of the currently sampled daughter asteroid (Fig. 4). Our model draws together some aspects of previous models of ureilites (Herrin et al., 2010; Warren, 2012; Horstmann and Bischoff, 2014; Goodrich et al., 2015; Sanders et al., 2017) but differs in the building blocks and the consequent radial compositional gradients within the original planetesimal. Our model also implies that the ureilite planetesimal accreted in a region of the solar nebula where primitive materials resembling $\mathrm{MgO}$-rich and $\mathrm{FeO}$-rich chondrules were abundant. Basalt dissipative magmatism which was common on all 



Figure 3 (a-c) Elemental ratios vs. $\Delta^{17} \mathrm{O}$ for metal-free bulk rock compositions of ureilites from Warren (2012) reconstructed ureilite bulk rock compositions, and a variety of primitive Solar System material (Table S-1).

C-rich asteroids that were roughly $150 \pm 50 \mathrm{~km}$ in diameter (Warren and Kallemeyn, 1992), is an integral feature of our model, and supports an original ureilite parent body that was at least $150 \pm 50 \mathrm{~km}$ in size.

The first stage (Fig. 4a) was accretion of a mixture of $\mathrm{MgO}$ rich chondrules with low $\Delta^{17} \mathrm{O}$ values, and FeO-rich chondrules with higher $\Delta^{17} \mathrm{O}$ values, the chondrules being used as proxy for the real primitive material that formed the original ureilite planetesimal. This mixture accounts both for the oxygen isotope variation (Fig. 1a) and the co-variation of composition with oxygen isotopes (Fig. 1b). The model is supported by observations of $\mathrm{FeO}-$ and $\mathrm{MgO}$-rich chondrules in single meteorites (Ruzicka, 2012). In our model, the first accreted material would have had a more $\mathrm{MgO}$-rich composition, with later material being more $\mathrm{FeO}$-rich, thereby producing a compositional gradient in the original ureilite planetesimal. Our model agrees with the two component model of Barrat et al (2017) based on carbon isotopes, and predicts that the high $\mathrm{MgO}$ chondrules would have low $\delta^{13} \mathrm{C}$ values, whereas the high $\mathrm{FeO}$ chondrules would have higher $\delta^{13} \mathrm{C}$ values. Although it does not preclude the possibility that ureilites could be derived from two or more parent bodies, our model clearly demonstrates that a compositionally zoned, single parent body (Downes et al., 2008) can successfully explain the observed geochemical and oxygen isotope signatures of ureilites.

Goodrich et al. (2001) showed that ${ }^{26} \mathrm{Al}$ was present in ureilites, although in amounts insufficient to cause complete melting (Budde et al. 2015). This fits well with the ages of chondrule formation, as Kurahashi et al. (2008) showed that $\mathrm{MgO}$-rich chondrules have ages of 1.7 to $2.5 \mathrm{Myr}$ after CAIs, whereas
FeO-rich chondrules have slightly younger ages (1.9 to 2.6 Myr after CAIs). Heating from ${ }^{26} \mathrm{Al}$ would have caused a rapid increase in temperature throughout the planetesimal leading to formation of an Fe sulfide melt (Fig. 4b) which coalesced to form a core. This removed siderophile and chalcophile elements, causing the depletion seen in remaining ureilite metals and bulk rocks (Rankenburg et al., 2008; Goodrich et al., 2013), and would also lead to a heavy Fe isotope composition of the silicate portion, consistent with investigations of ureilites, iron meteorites and experimental observations (Williams et al., 2006; Schuessler et al., 2007; Budde et al., 2015).

Heating continued until silicate partial melting began. Temperatures varied from $1320^{\circ} \mathrm{C}$ in the high $\mathrm{MgO}$ inner part of the planetesimal to $1190{ }^{\circ} \mathrm{C}$ in the low $\mathrm{MgO}$ outer part (Warren, 2012). Silicate melts, including trachyandesites and basalts, were formed by fractional melting in all silicate portions of the planetesimal (Fig. 4c). This accounts for REE-depletion in the residual ureilites (Guan and Crozaz, 2001). These silicate melts are found as melt inclusions and melt clasts (Goodrich et al., 2001; Cohen et al., 2004). Some melts may have intruded into the ureilite mantle and contributed to formation of pyroxene-rich ureilites (Downes et al., 2008). Formation and rapid removal of silicate melts formed from low degrees of partial melting would not have homogenised the major element radial compositional gradients within the planetesimal interior.

Silicate melting removed ${ }^{26} \mathrm{Al}$ from the planetesimal interior, so cooling began. However, while the planetesimal was still hot, it was disrupted by a major impact (Fig. 4d; Warren and Kallemeyn, 1989; Herrin et al., 2010). During this event, hot olivines and pyroxenes reacted with carbon and 


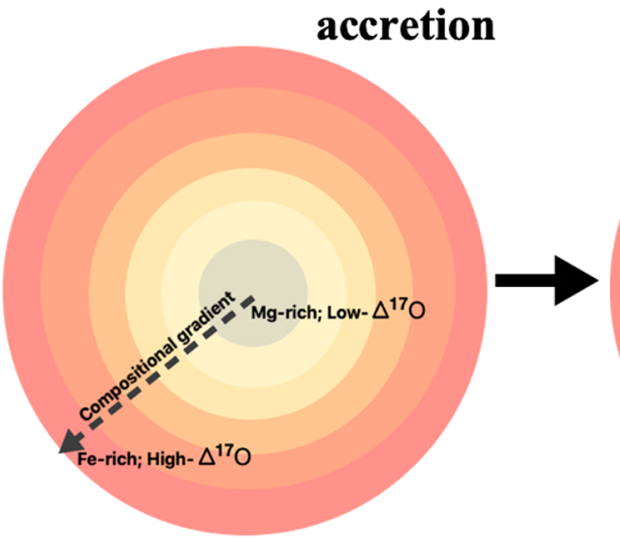

(a)

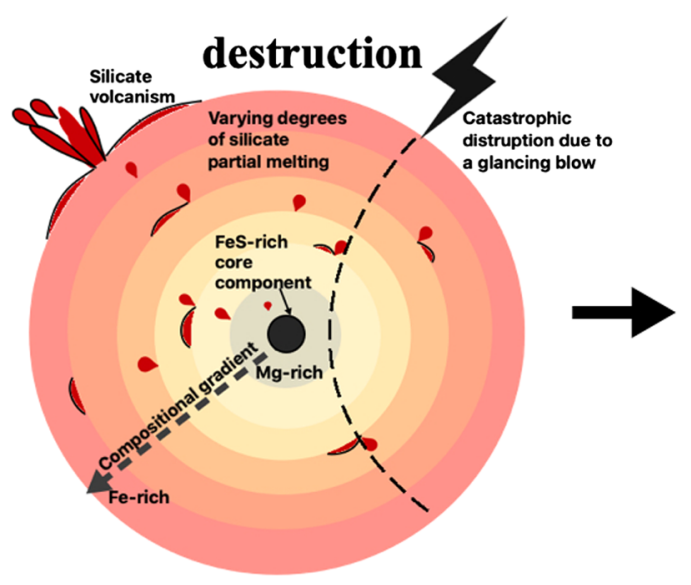

(d)

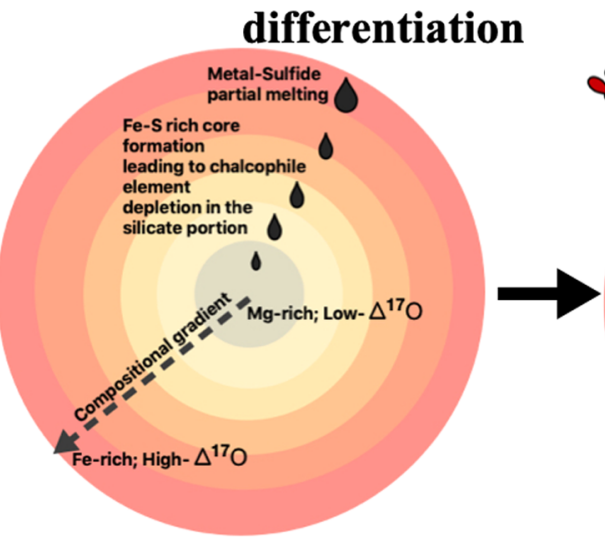

(b)

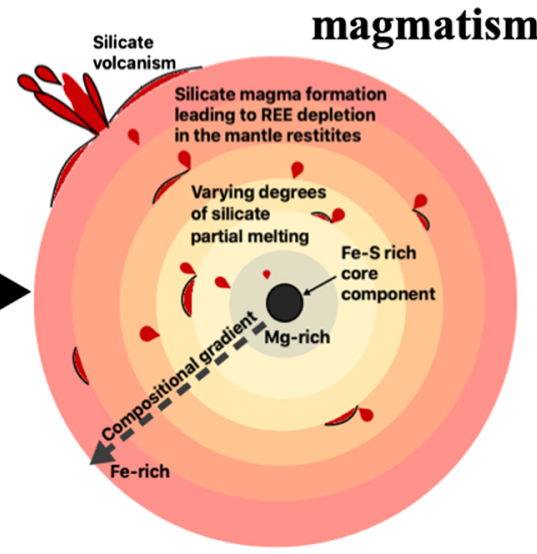

(c)

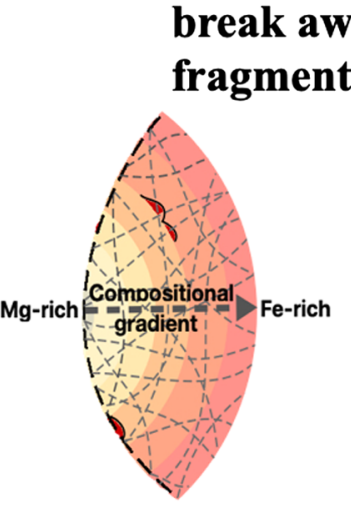

(e)



jumble pile daughter asteroid

Figure 4 Model of stages in history of ureilite planetesimal. (a) Accretion of MgO-rich and FeO-rich chondrules; MgO-rich chondrules accrete slightly earlier, leading to a compositional gradient within the growing planetesimal. (b) Heating and formation of eutectic metal-sulfide melt, migration of melt and coalescence to form a core. (c) Silicate partial melting and removal of magma. (d) Disruption of planetesimal by a glancing blow from an impactor; more of the shallow FeO-rich material was removed from the planetesimal than the deeper MgO-rich material, and the S-rich core was not disrupted. (e) Fractured breakaway portion. (f) Re-accreted present day ureilite asteroid composed of jumbled fragments of the breakaway portion. Darker shading=FeO-rich ureilites; lighter shading $=$ MgO-rich ureilites; red = silicate melt-enriched material.

formed the characteristic reverse zoned olivines decorated with metallic iron blebs. To account for the distribution of olivine compositions (Fig. 3), disruption must have been caused by a glancing blow, such that the outer Fe-rich portions were preferentially disrupted (Fig. 4d,e). The S-rich core remained intact, together with the remaining $\mathrm{MgO}$-rich inner silicate portion of the planetesimal. A daughter asteroid (the present day ureilite parent body) then re-accreted from the jumbled and chaotic silicate fragments (Fig. 4f; Herrin et al., 2010; Goodrich et al., 2015), from which present day ureilites are derived.

\section{Conclusions}

Many early Solar System processes are exemplified by ureilite meteorites. We have used constraints from the geochemical trends displayed by ureilites (Fig. 1), and their reconstructed bulk major element compositional range (Fig. 3), to produce a two component model consisting of $\mathrm{MgO}$-rich and $\mathrm{FeO}$-rich chondrules, that have been used in our compositional model as proxy for the real primitive material that formed the original ureilite planetesimal. This successfully explains the correlation between oxygen isotopes and Fo contents of olivine cores in ureilites. The model also explains the trace element systematics of the siderophile and rare earth elements, by formation of an FeS core and silicate melts, respectively.

\section{Acknowledgements}

We gratefully acknowledge the Leverhulme Trust for financial support (RPG2012-711), and the Natural History Museum for support. Noriko Kita, Sara Russell, Ian Sanders, Ed Scott and Cyrena Goodrich provided useful feedback. The manuscript improved from the constructive reviews and thoughtful suggestions by two anonymous reviewers and the editor.

\section{Editor: Helen Williams}

\section{Additional Information}

Supplementary Information accompanies this letter at http:// www.geochemicalperspectivesletters.org/article2018.

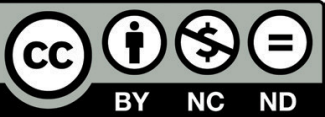

(C) 2020 The Authors. This work is distributed under the Creative Commons Attribution NonCommercial No-Derivatives 4.0 
License, which permits unrestricted distribution provided the original author and source are credited. The material may not be adapted (remixed, transformed or built upon) or used for commercial purposes without written permission from the author. Additional information is available at http://www. geochemicalperspectivesletters.org/copyright-and-permissions.

Cite this letter as: Rai, N., Downes, H., Smith, C. (2020) Ureilite meteorites provide a new model of early planetesimal formation and destruction. Geochem. Persp. Let. 14, 20-25.

\section{References}

Barrat, J.-A., Rouxel, O., Wang, K., MoYnier, F., Yamaguchi, A., Bischoff, A LANGLADE, J. (2015) Early stages of core segregation recorded by Fe isotope in an asteroidal mantle. Earth and Planetary Science Letters 419, 93-100.

Barrat, J.-A., Sansjofre, P., Yamaguchi, A., Greenwood R.C., Gillet, P. (2017) Carbon isotopic variation in ureilites: Evidence for an early, volatile-rich Inner Solar System. Earth and Planetary Science Letters 478, 143-149.

Bischoff, A., Horstmann, M., Barrat, J.-A., Chaussidon, M., Pack, A., Herwartz, D. Ward, D., Vollmer, C., DeCKer, S. (2014) Trachyandesitic volcanism in the early Solar System. Proceedings of the National Academy of Science 111, 12689-12692.

Budde, G., Kruijer, T.S., Fisher-Gödde, M., Irving, A.J., Kleine, T. (2015) Planetesimal differentiation revealed by the Hf-W systematics of ureilites. Earth and Planetary Science Letters 430, 316-325.

Clayton, R.N., MAYEDA, T.K. (1988) Formation of ureilites by nebular processes Geochimica et Cosmochimica Acta 52, 1313-1318.

Cohen, B., Goodrich, C.A., KeIL, K. (2004) Feldspathic clast populations in polymict ureilites: Stalking the missing basalts from the ureilite parent body. Geochimica et Cosmochimica Acta 68, 4249-4266.

Downes, H., Mittlefehldt, D.W., Kita, N.T., Valley, J.W. (2008) Evidence from polymict ureilite meteorites for a disrupted and re-accreted single ureilite parent asteroid gardened by several distinct impactors. Geochimica et Cosmochimica Acta 72, 4825-4844.

Elkins-Tanton, L.T. (2012) Magma Oceans in the Inner Solar System. Annual Reviews of Earth and Planetary Science 40, 113-139.

Goodrich, C.A., FioretTi, A.M., Tribaudino, M., Molin, G. (2001) Primary trapped melt inclusions in olivine in the olivine-augite-orthopyroxene ureilite Hughes 009. Geochimica et Cosmochimica Acta 65, 621-652.

GoodRICH, C.A., ScotT, E.R.D., FIORETTI, A.M. (2004) Ureilitic breccias: clues to the petrological structure and impact disruption of the ureilite parent asteroid. Chemie der Erde 64, 283-327.

GoOdrich, C.A., Ash, R.D, Van Orman, J.A, Domanik, K, McDonough, W.F. (2013) Metallic phases and siderophile elements in main group ureilites: Implications for ureilite petrogenesis. Geochimica et Cosmochimica Acta $112,340-373$

Goodrich, C.A., Hartmann, W.K., O’Brien, D.P., Wiedenschilling, S.J., Wilson, L Michel P., JutZI, M. (2015) Origin and history of ureilitic material in the solar system: The view from asteroid $2008 \mathrm{TC}_{3}$ and the Almahata Sitta meteorite. Meteoritics and Planetary Science 50, 782-809.

GuAn, Y., CrozAZ, G. (2001) Microdistributions and petrogenetic implications of rare earth elements in polymict ureilites. Meteoritics and Planetary Science 36, 1039-1956.

Hayden, L.A., Van Orman, J.A., McDonough, W.F., Ash, R.D., Goodrich, C.A. (2011) Trace element partitioning in the Fe-S-C system and its implication for planetary differentiation and the thermal history of ureilites. Geochimica et Cosmochimica Acta 75, 6570-6583.

Herrin, J.S., Zolensky, M.E., Ito, M., Le, L., Mittlefehldt, D.W., Jenniskens, P., Ross, A.J., SHADDAD, M.H. (2010) Thermal and fragmentation history of ureilitic asteroids: Insights from the Almahata Sitta fall. Meteoritics \& Planetary Science 45, 1789-1803

Horstmann, M., Bischoff, A. (2014) The Almahata Sitta polymict breccia and the late accretion of asteroid 2008TC 3 . Chemie der Erde 74, 149-183.

Jenniskens, P., Shaddad, M.H., Numan, D., Elsir, S., Kudoda, A.M., et al. (2009) The impact and recovery of asteroid $2008 \mathrm{TC}_{3}$. Nature 458, 485-488.

Kurahashi, E., Kita, N.T., Nagahara, H., Morishita, Y. (2008) ${ }^{26} \mathrm{Al}-{ }^{26} \mathrm{Mg}$ systematics of chondrules in a primitive $\mathrm{CO}$ chondrite. Geochimica et Cosmochimica Acta 72, 3865-3882.

Li, S., Yin, Q.Z., BaO, H., Sanborn, M.E., Irving, A., Ziegler, K., Agee, C., Marti, K., MiAO, B., LI, X., LI, Y., WANG, S. (2018) Evidence for a multi-layered internal structure of the chondritic acapulcoite-lodranite parent asteroid. Geochimica et Cosmochimica Acta 242, 82-101.

LiboureL, G., KROT, A.N. (2007) Evidence for the presence of planetesimal material among the precursors of magnesian chondrules of nebular origin. Earth Planetary Science Letters 254, 1-8.

Mittlefehldt, D.W., McCoy, T.J., Goodrich, C.A., Kracher, A. (1998) Nonchondritic meteorites from asteroidal bodies. In: PAPIKE, J.J. (Ed.) Planetary Materials. Reviews in Mineralogy 36. Mineralogical Society of America, Washington, D.C., 4-1-4-195.

Rankenburg, K., Humayun, M., Brandon, A.D., Herrin, J.S. (2008) Highly siderophile elements in ureilites. Geochimica et Cosmochimica Acta 72, 4642-4659.

RuzicKA, A. (2012) Chondrule formation by repeated evaporative melting and condensation in collisional debris clouds around planetesimals. Meteoritics and Planetary Science 47, 2218-2236.

Sanders, I.S., Scott, E.R.D., Delaney, J.S. (2017) Origin of mass-independent oxygen isotope variation among ureilites: Clues from chondrites and primitive achondrites. Meteoritics and Planetary Science 52, 690-708.

ScotT, E.R.D., TAYLOR, G.J., KeIL, K. (1993) Origin of ureilite meteorites and implications for planetary accretion. Geophysical Research Letters 20, 415-418.

Schuessler, J.A., Schoenberg, R., Behrens, H., von Blanckenburg, F. (2007) The experimental calibration of the iron isotope fractionation factor between pyrrhotite and peralkaline rhyolitic melt. Geochimica et Cosmochimica Acta 71, 417-433.

SingLETARY, S., Grove, T.L. (2006) Experimental constraints on ureilite petrogenesis. Geochimica et Cosmochimica Acta 70, 1291-1308.

WARren, P.H. (2012) Parent body depth-pressure-temperature relationships and the style of the ureilite anatexis. Meteoritics and Planetary Science 47, 209-227.

Warren, P.H., Kallemeyn, G.W. (1989) Geochemistry of polymict ureilite EET 83309, and a partially-disruptive impact model for ureilite origin. Meteoritics 24, 233-246.

WarRen, P.H., KallemeYN, G.W. (1992) Explosive volcanism and the graphiteoxygen fugacity buffer on the parent asteroid of the ureilite meteorites. Icarus 100, 110-126.

Warren, P.H., Ulff-Moller, F., Huber, H., Kallemeyn, G.W. (2006) Siderophile geochemistry of ureilites: A record of early stages of planetesimal core formation. Geochimica et Cosmochimica Acta 70, 2104-2126.

Williams, H.M., Markowskia, A., Quittéa, G., Halliday, A.N., Teutscha, N., LeVASSEUR, S. (2006) Fe isotope fractionation in iron meteorites: New insights into metal-sulphide segregation and planetary accretion. Earth and Planetary Science Letters 250, 486-500. 